\title{
EVIDÊNCIAS EMPÍRICAS SOBRE CONVERGÊNCIA DA DESIGUALDADE DE RENDA ENTRE OS MUNICÍPIOS CEARENSES
}

\author{
Airton Lopes Amorim \\ Universidade Federal de Viçosa (UFV) \\ Ricardo Bruno Nascimento dos Santos \\ Universidade Federal do Pará (UFPA) \\ Eliane Pinheiro de Sousa \\ Universidade Regional do Cariri (URCA) \\ Daniel Arruda Coronel \\ Universidade Federal de Santa Maria (UFSM)
}

EVIDÊNCIAS EMPÍRICAS SOBRE CONVERGÊNCIA DA DESIGUALDADE DE RENDA ENTRE OS MUNICÍPIOS CEARENSES

Resumo: A desigualdade de renda tende a diminuir em municipios com elevada desigualdade e a aumentar naqueles com baixa desigualdade? Este trabalho tenta responder a essa questão ao verificar se existiu convergência da desigualdade de renda entre os municípios cearenses, nos anos 1991 e 2000. A principal medida de desigualdade de renda utilizada foi o índice de Gini, sendo os testes de convergência realizados por meio de modelos com efeito threshold, nos quais as variáveis concernentes ao índice de Gini, à renda per capita e aos anos de estudo, medidas no período inicial, foram consideradas como possíveis variáveis threshold. Os resultados permitiram rejeitar a hipótese de clubes de convergência da desigualdade de renda entre os municípios cearenses. No entanto, não se pode rejeitar a hipótese de convergência condicional da desigualdade de renda entre os mesmos, sendo que eles estariam convergindo para um valor médio de equilíbrio de desigualdade de renda maior, ou seja, os municípios cearenses estariam tornando-se mais concentradores de renda per capita.

Palavras-chave: Desigualdade de renda, Índice de Gini, efeitos Threshold.

\section{EMPIRICAL EVIDENCES ABOUT THE CONVERGENCE OF INCOME INEQUALITY AMONG CITIES FROM CEARA}

Abstract: The income inequality tends to decrease in municipalities with high inequality and increase in those with low inequality? This paper intends to answer this question by checking if there was convergence of income inequality in the municipalities of the State of Ceará, in the years 1991 and 2000. The main measurement of income inequality used was the Gini index, with the convergence tests conducted through models with threshold effect, in which the variables relating to the Gini index, to the per capita income and to the years of study were considered, measured in the initial period as possible threshold variables. The results allowed rejecting the hypothesis of convergence clubs of the per capita income inequality among the cities from Ceará. However there is no way to reject the hypothesis of conditional convergence of the income inequality in the municipalities among the same, where these would be converging to an average value of the bigger income inequality, that is, they would be turning themselves into more per capita incomeconcentrating municipalities.

Key words: Income inequality, Gini Index, Threshold effect. 


\section{INTRODUÇÃO}

Entre 1991 e 2000, o estado do Ceará registrou um acréscimo de 17,085\% no Índice de Desenvolvimento Humano (IDH), que passou de 0,597, em 1991, para 0,699 em 2000. Além disso, o Estado também apresentou um crescimento de $71,58 \%$ no PIB per capita entre 2003 e 2008, que aumentou de $R \$ 4.145,00$, em 2003, para $R \$ 7.112,00$ em 2008. No entanto, não obstante esses bons resultados, o Ceará possui elevada desigualdade de renda (INSTITUTO DE PESQUISA E ESTRATÉGICA ECONÔMICA DO CEARÁ, 2010).

Dados do Atlas do Desenvolvimento Humano, um trabalho conjunto do Programa das Nações Unidas para o Desenvolvimento (PNUD), Instituto de Pesquisa Econômica Aplicada (IPEA) e Fundação João Pinheiro (FJP) (2003) mostram que o grau de desigualdade existente na distribuição da renda per capita no estado do Ceará, medida pelo índice de Gini, cresceu entre 1991 e 2000, passando de 0,654 para 0,675 , constituindo-o como o Estado da região Nordeste com o segundo pior valor para o índice de Gini em 2000, ficando atrás apenas de Alagoas.

Infelizmente, o estado do Ceará não se destaca apenas pela elevada desigualdade na distribuição de renda. Como observado por Alcântara (2003 apud SOUSA, 2009), são perceptíveis as enormes desigualdades entre as várias regiões cearenses. A ocupação demográfica e econômica do território cearense, por exemplo, é extremamente desbalanceada, com a Região Metropolitana de Fortaleza (RMF), que ocupa apenas $3,5 \%$ do espaço estadual, abrigando, em 2003, $42 \%$ da população e concentrando cerca de $62 \%$ do PIB e $90 \%$ da arrecadação de tributos. Verifica-se, ainda, que apesar dos municípios cearenses localizados no interior do Estado empregarem $61 \%$ da força de trabalho do Estado, cobrindo quase todo o setor agrícola e perto da metade da indústria e dos serviços, o Produto Interno Bruto (PIB) obtido por esses municípios atinge somente $38 \%$ do PIB do Estado. Como resultado, o PIB per capita desses municípios interioranos atinge $42 \%$ do PIB per capita da RMF.
O baixo nível de renda nos municípios do interior, com todas as suas implicações para a política de desenvolvimento, aponta para uma situação em que $89 \%$ dos impostos coletados pelo estado, em 1999, originavamse da RMF, com o Interior contribuindo com uma baixa e desproporcionada parcela de $11 \%$. Essa desigualdade de renda entre os municípios mais industrializados, como Eusébio, Horizonte, Maracanaú, que fazem parte da RMF, e o interior do Estado também foi verificada por Barreto (2007).

Como o estado do Ceará tem sido caracterizado por elevada desigualdade de renda, estudos sobre essa questão têm ocupado um espaço substancial nas agendas de pesquisa. 0 estudo desenvolvido por Mayorga, Tabosa e Amaral Filho (2007), por exemplo, avaliou a desigualdade de renda no estado do Ceará, no período de 1997-2002, mediante as relações existentes entre rendas per capita, índice de Theil e coeficiente de variação ponderado de Williamson. Silva e Loureiro (2007) procuraram apontar evidências mais detalhadas sobre a tendência de crescimento da desigualdade de renda no Ceará, no período entre 2001 e 2005. Soares (2008), por sua vez, analisou a desigualdade de renda a partir do emprego formal para os anos de 1999 a 2005, utilizando índice de Gini, índice de desigualdade Theil-T e de desigualdade Theil-L.

A questão apreciada neste trabalho diz respeito ao comportamento da desigualdade de renda per capita entre os municípios cearenses, fundamentada, por sua vez, nos estudos citados anteriormente, que constataram o aumento da desigualdade de rendaneste Estado eque destacaram a disparidade regional dentro do mesmo. Em outros termos, buscou-se verificar se a desigualdade de renda tenderia a diminuir nos municípios cearenses com elevada desigualdade e aumentar naqueles com baixa desigualdade, como apontado por algumas versões mais recentes dos modelos neoclássicos de crescimento (AYAGARI, 1991; BERTOLA, 1995; BÉNABOU, 1996). 
0 estudo foi realizado por meio da aplicação de dois testes concorrentes de convergência da desigualdade da renda domiciliar per capita, a saber, testes de clube de convergência e testes de convergência condicional, nos municípios cearenses, nos anos 1991 e 2000. Esses testes foram realizados por meio da estimação de modelos econométricos com efeito threshold, considerando o índice de Gini como principal medida de desigualdade de renda ${ }^{1}$. De maneira geral, pôdese concluir pela não aceitação da hipótese de clube de convergência, mas pela aceitação da hipótese de convergência condicional. Ademais, o valor médio de longo prazo do índice de Gini foi maior do que o valor médio desse índice medido em 2000, indicando que a desigualdade de renda nos municípios do Ceará tornar-se-ia mais elevada.

Este trabalho está estruturado em quatro seções, além desta introdução. Na segunda, expõese a fundamentação teórica do trabalho; na terceira, a metodologia e a fonte de dados do trabalho; na quarta, os resultados obtidos são analisados e discutidos e, finalmente, são apresentadas algumas considerações sobre o estudo.

\section{EVIDÊNCIAS EMPÍRICAS SOBRE A CONVERGÊNCIA DA DESIGUALDADE DE RENDA}

A revisão dos trabalhos empíricos sobre convergência da desigualdade de renda mostra que os mesmos são bem recentes na literatura de crescimento econômico. De acordo com Ravallion (2001) Bénabou (1996) parecer ter sido o primeiro a testar a hipótese da convergência da desigualdade de renda, encontrando evidências desse processo por meio de diferentes conjuntos de dados cross section de países, para os anos 1970 e 1990.

Posteriormente, essa hipótese foi confirmada pelo próprio Ravallion (2001; 2003), por meio de um conjunto de dados internacionais mais recentes, 1985 a 1995. Ravallion encontrou que a desigualdade de renda per capita e do consumo per capita entre os países, quando medidas pelo índice de
Gini, estaria convergindo para seus niveis médios um índice de aproximadamente $40 \%$. Ainda de acordo com esse autor, os resultados seriam robustos para erros nas medidas de desigualdade e para a dinâmica de curto prazo ao longo de sua tendência de longo prazo. Entretanto, 0 autor pondera seus resultados ao considerar que o processo de convergência se daria de forma lenta, sendo necessárias mais observações ao longo do tempo para confirmar esses resultados.

Bleaney e Nishiyama (2003) verificaram que o processo de convergência da desigualdade de renda difere entre países desenvolvidos e em desenvolvimento, com a velocidade de convergência sendo maior nos países avançados. Os resultados encontrados por esses autores, para os anos 1965 e 1990, indicaram que a convergência da desigualdade de renda parece estar se firmando mais significativamente entre os países da Organização para a Cooperação e Desenvolvimento Econômico (OCDE), do que entre os países em desenvolvimento. No entanto, devido à limitação da amostra de países utilizados, ainda não estaria claro se haveria convergência de maneira geral, mas onde foi possível verificar esse processo, os países em desenvolvimento parecem estar convergindo para uma distribuição de renda menos igualitária do que os países da OCDE.

Ezcurra e Pascual (2005) examinaram a distribuição regional da desigualdade de renda na União Europeia entre 1993 e 1998. Os resultados obtidos revelam a existência de um processo de convergência dos níveis de desigualdade regional ao longo do período analisado. De acordo com esses autores, esse processo ocorreu pela redução da dispersão de renda nas regiões que registraram níveis relativamente elevados de desigualdade em 1993. A polarização na distribuição de renda diminuiu independentemente do número de grupos considerados, no entanto, uma vez que o nível observado de mobilidade entre grupos é baixo, as regiões europeias tendem, ao longo do tempo, a manter suas posições relativas em termos de desigualdade. 
Panizza (2001), utilizando dados da economia dos Estados Unidos, inaugurou uma nova etapa na análise da convergência da desigualdade de renda, ou seja, a busca de evidências dentro de países, encontrado-as.

Considerando o enfoque de Panizza (2001), podem-se destacar alguns trabalhos que procuraram investigar a hipótese da convergência da desigualdade da renda per capita dentro do Brasil, tendo como foco de análise os municípios brasileiros, para os anos 1991 e 2000.

Gomes (2007), por exemplo, investigou a hipótese de convergência da desigualdade de renda entre os municípios brasileiros. Conforme enfatizado por esse autor, ao restringir sua análise a um único país e controlar diferenças regionais, é possível interpretar os testes empíricos como testes de convergência condicional. Dessa forma, os resultados indicam que os municípios brasileiros estariam convergindo para um nível de desigualdade de longo prazo maior do que 0 atual (medido no ano de 2000), com exceção para os municípios localizados nos estados da Região Sul.

Ferreira e Santos da Cruz (2008), por sua vez, mostraram que há evidências empíricas de que 0 processo de clubes de convergência da desigualdade de renda entre os municípios brasileiros esteja ocorrendo, identificando seis clubes a partir do índice de Gini inicial. Dessa forma, dependendo do nível inicial de desigualdade, observa-se a ocorrência de múltiplos estados estacionários para os municípios brasileiros, cada um deles formando um clube de convergência com características socioeconômicas distintas. Em todos os clubes, a renda do trabalho e a renda proveniente das transferências governamentais apresentaram correlação negativa com a taxa de crescimento do índice de Gini, calculada entre os anos 1991 e 2000, sendo a elasticidade da renda do trabalho sempre superior à da renda de transferências.

Este trabalho procurou fornecer estimativas para um nivel geográfico mais concentrado, ou seja, o Estado do Ceará. É preciso esclarecer que essa abordagem empírica não se trata apenas de explorar uma sequência natural de divisão político regional. Se for considerada a extensão territorial do Brasil, bem como o processo histórico de ocupação e exploração econômica das regiões de nosso país, é evidente que as políticas econômicas que visem à redução das desigualdades regionais (em qualquer aspecto econômico) devem levar em consideração as especificidades de cada estado brasileiro.

\section{ABORDAGEM EMPÍRICA DOS TESTES DA CONVERGÊNCIA DA DESIGUALDADE DE RENDA}

0 teste mais direto para convergência da desigualdade da renda per capita entre países, regiões, estados ou municípios foi inspirado na literatura empírica sobre crescimento econômico e baseia-se na estimação de uma equação de regressão para verificar a existência de correlação entre as mudanças percentuais de desigualdade de renda entre dois períodos e o nível inicial dessa variável ${ }^{2}$.

Vale observar que no caso de clubes de convergência, as diferenças na desigualdade de renda per capita refletem países ou regiões que se situam em bases de atração distintas (definidas pelas condições iniciais), sendo que a estratégia econometria adequada para esse caso é a divisão dos países ou regiões entre grupos, usando variáveis que reflitam suas condições iniciais. Assim, cada país pertencerá a uma zona de atração determinada por suas condições iniciais, com cada uma delas caracterizada por um estado estacionário único, para o qual todos os membros convergem no longo prazo. A pressuposição por trás desse teste é a existência de múltiplos equilíbrios de estado estacionário, localmente estáveis (GALOR, 1996).

No caso da convergência condicional, as diferenças na desigualdade de renda per capita refletem heterogeneidades nos parâmetros, sendo que a estratégia econométrica apropriada para testála é a introdução de variáveis de controle na regressão de crescimento do índice de Gini para representar o estado estacionário de cada economia. 


\section{1 Clubes de convergência}

Para testar a hipótese de clubes de convergência da desigualdade da renda per capita entre os municípios cearenses, este trabalho baseouse em Hansen (2000), que sugeriu um procedimento bootstrap para testar a hipótese de uma formulação linear contra a formulação threshold alternativa.

O modelo threshold assume que os dados são gerados pelos valores $\left\{y_{i}, x_{i}, q_{i}\right\}_{i=1}^{n}$, em que $y_{i} \mathrm{e}$ $q_{i}$ são observações sobre a variável dependente e a variável threshold, respectivamente, e $x_{i}$ é um vetor $p X 1$ de variáveis independentes para cada unidade observada. A variável threshold $q_{i}$ divide a amostra em diferentes grupos, clubes, classes ou regimes, sendo que a mesma pode fazer parte de $x_{i}$.

0 modelo de regressão com efeito threshold pode ser expresso como:

$$
\begin{array}{ll}
y_{i}=\beta_{1} x_{i}+e_{i}, & q_{i} \leq \gamma(1) \\
y_{i}=\beta_{2} x_{i}+e_{i}, & q_{i}>\gamma(2)
\end{array}
$$

com o valor $\gamma$ dividindo a amostra em dois grupos. A variável aleatória $e_{i}$ é o termo de erro da regressão.

0 modelo acima pode ser reescrito em uma única equação, bastando para isso, que se defina uma variável dummy $d_{i}(\gamma)=I\left\{q_{i} \leq \gamma\right\}$, em que $I\{\}\}$ é uma função indicadora. Considerandose $x_{i}(\gamma)=x_{i} d_{i}(\gamma)$, as equações (1) e (2) podem ser expressas como:

$$
y_{i}=x_{i} \beta+x_{i}(\gamma) \theta+e_{i}(3)
$$

em que: $\beta=\beta_{2}$ e $\theta=\beta_{1}-\beta_{2}$. A equação (3)

permite que os coeficientes da regressão diferenciemse entre os grupos da amostra.

Para encontrar o valor de $\hat{\gamma}$ que minimiza a equação (3), deve-se realizar uma análise sobre os valores de $\gamma$, tal que $\hat{\gamma}=\operatorname{argmin} S_{n}(\gamma)$, em que $S_{n}(\gamma)$ é função de soma de qualứr ${ }^{n}$ dos dos erros. Após a determinação de $\hat{\gamma}$, as estimativas de Mínimos Quadrados Ordinários de $\theta$ e $\beta$ são dadas por $\hat{\theta}=\hat{\theta}^{\prime}(\hat{\gamma})$ e $\hat{\beta}=\hat{\beta}(\hat{\gamma})$.

Uma hipótese importante para o teste dos clubes de convergência é se o modelo threshold é estatisticamente significativo em relação à especificação linear simples. Nesse caso, a hipótese nula descreve uma especificação linear simples e pode ser expressa como:

$$
H_{0}: \beta_{1}=\beta_{2}(4)
$$

Testes da hipótese nula em (4) incorrem em algumas dificuldades, uma vez que o parâmetro threshold y não é identificado sob essa hipótese. No entanto, Hansen (2000) sugeriu um procedimento bootstrap do teste do Multiplicador de Lagrange (LM), consistente com heterocedasticidade, para testar a hipótese nula de uma formulação linear contra a alternativa threshold. A estratégia utilizada por Hansen é baseada na estatística de verossimilhança $B_{n}(\gamma)$.

Para elaborar regiões de confiança baseadas em $B_{n}(\gamma)$, define-se $C$ como 0 nível de intervalo de confiança assintótico $(C=0,9)$ e $c=c_{\xi}(C)$ como 0 valor crítico do intervalo $\hat{\Gamma}=\left\{\gamma: \mathbb{R}_{n}(\gamma) \leq c\right\}$.

Observa-se que, se a hipótese de homocedasticidade é rejeitada, a estatística de verossimilhança é redefinida como:

$$
\mathbb{R}_{n}^{*}(\gamma)=\frac{R_{n}(\gamma)}{\hat{\eta}^{2}}=\frac{S_{n}(\gamma)-S_{n}(\hat{\gamma})}{\hat{\sigma}^{2} \hat{\eta}^{2}}(5)
$$

\subsection{Convergência condicional}

Caso a hipótese de clubes de convergência seja rejeitada, tem-se a possibilidade de testar a hipótese de convergência condicional. Assim, considera-se a amostra como um todo, ou seja, não há a separação da mesma em grupos ou regimes diferenciados e estima-se, por Mínimos Quadrados Ordinários, a seguinte equação de regressão:

$$
y_{i}=\beta_{i} x_{i}+e_{i},(6)
$$

em que $y_{i}$ representa a variável dependente; $x_{i}$ é um vetor $p X 1$ de variáveis independentes, as mesmas consideradas nas equações (1) e (2), para cada unidade observada e $e_{i}$ é o termo de erro da regressão.

\subsection{Modelo empírico}

As hipóteses de convergência da desigualdade de renda entre os municípios cearenses 
foram testadas por meio da seguinte equação de regressão, baseada no trabalho de Ferreira e Santos da Cruz (2008)

$$
\begin{aligned}
& \int \mathrm{h}\left[\frac{\text { Gini }_{i, t}}{\text { Gini }_{i,-1}}\right]=\left\{\beta_{0}^{1}+\beta_{1}^{1} \mathrm{~h}\left(\text { Gini }_{i, t-1}+\beta_{2}^{1} \mathrm{~h}\left(\frac{\text { Rtrab }_{t}}{\text { Rtrab }_{t-1}}\right)_{i}+\beta_{3}^{1} \mathrm{~h}\left(\frac{\text { Rtransf }_{t}}{\text { Rtrans }_{t-1}}\right)_{i}\right.\right. \\
& \left.+\beta_{4}^{1} \mathrm{~h}\left(\frac{\text { Estudo }_{t}}{\text { Estudo }_{t-1}}\right)_{i}+\beta_{5}^{1} \mathrm{~h}\left(\frac{\text { Popadul }_{t}}{\text { Popadult }_{t-1}}\right)_{i}\right\}+u_{i} \quad \boldsymbol{B}\left(\text { Gini }_{t-1} \leq \gamma\right) \\
& \mathrm{h}\left[\frac{\operatorname{Gini}_{i, t}}{\operatorname{Gin}_{i, t-1}}\right]=\left\{\beta_{0}^{2}+\beta_{1}^{2} \mathrm{~h}\left(\text { Gini }_{i, t-1}+\beta_{2}^{2} \mathrm{~h}\left(\frac{\text { Rtrab }_{t}}{\text { Rtrab }_{t-1}}\right)_{i}+\beta_{3}^{2} \mathrm{~h}\left(\frac{\text { Rtrans }_{t}}{\text { Rtrans }_{t-1}}\right)_{i}\right.\right. \\
& \left.+\beta_{4}^{2} \mathrm{~h}\left(\frac{\text { Estudo }_{t}}{\text { Estudo }_{t-1}}\right)_{i}+\beta_{5}^{2} \mathrm{~h}\left(\frac{\text { Popadul }_{t}}{\text { Popadult }_{t-1}}\right)\right\}_{i}+u_{i} \quad \boldsymbol{E}\left(\text { Gini }_{t-1}>\gamma\right)
\end{aligned}
$$

em que:

a) Índice de Gini: mede o grau de desigualdade existente na distribuição de indivíduos segundo a renda domiciliar per capita. Seu valor varia de 0 , quando não há desigualdade (a renda de todos os indivíduos tem o mesmo valor), a 1, quando a desigualdade é máxima (apenas um indivíduo detém toda a renda da sociedade, e a renda de todos os outros indivíduos é nula).

b) Renda do trabalho: participação percentual das rendas provenientes do trabalho (principal e outros) na renda total de cada município cearense;

c) Renda de transferências: participação percentual das rendas provenientes de transferências governamentais (aposentadorias, pensões e programas oficiais de auxílio, como renda mínima, bolsa-escola e seguro-desemprego etc.) na renda total de cada município cearense;

d) Anos de estudo: média dos anos de estudo de pessoas com 25 anos ou mais para cada município cearense;

e) População adulta: proporção de pessoas adultas (pessoas com 25 anos ou mais) para cada município cearense.

A evidência de convergência (divergência) está associada com o sinal negativo (positivo) de $\beta_{1}$ o parâmetro de convergência; nesse caso, as economias com menores valores do índice de Gini no período inicial apresentariam um crescimento mais acelerado dessa variável ao longo do período de análise, considerado em relação às economias com maiores valores do índice de Gini. Os clubes de convergência, por sua vez, são determinados a partir da aplicação do teste de linearidade ${ }^{4}$.

É importante destacar que este trabalho diferencia-se dos demais já realizados, em especial o trabalho de Ferreira e Santos da Cruz (2008), em dois aspectos fundamentais. Primeiro, a variável proporção de pessoas adultas foi incluída como variável de controle no modelo empírico. De acordo com o relatório sobre a recente queda da desigualdade de renda no Brasil, publicado pelo IPEA, em 2006, a variação das características demográficas das famílias seria um dos determinantes imediatos da mudança na desigualdade da renda domiciliar per capita, uma vez que a capacidade de geração de renda das famílias deve-se, entre outros fatores, ao número de pessoas adultas. Segundo, para testar a robustez dos resultados, foram utilizadas medidas alternativas de desigualdade da renda familiar per capita (índice de Theil - L, Razão entre a renda média dos $10 \%$ mais ricos e os $40 \%$ mais pobres, e a Razão entre a renda média dos $20 \%$ mais ricos e os $40 \%$ mais pobres) bem como diferentes variáveis como possível variável threshold.

\subsection{Fonte de dados}

A amostra utilizada para a estimação compreende 184 municípios do estado do Ceará, nos anos 1991 e 2000, anos do censo populacional. Os dados foram coletados no site do IPEA-DATA.

\section{ANÁLISE E DISCUSSÃO DOS RESULTADOS}

\subsection{Clubes de convergência da desigualdade de renda}

Para testar a hipótese de clubes de convergência da desigualdade da renda domiciliar 
per capita nos municípios cearenses, foi aplicado 0 teste do Multiplicador de Lagrange (LM) no modelo (7). Esse procedimento consiste em verificar se existe evidência para o efeito threshold, considerando a hipótese nula de inexistência desse efeito e computando os $p$-valores por meio da técnica bootstrap.

Foram utilizadas três variáveis para medir o possivel efeito threshold, a saber: índice de Gini, renda per capita e anos de escolaridade, todas medidas em relação ao período inicial, uma vez que os clubes de convergência dependem das condições iniciais das economias em análise. Nesse modelo, a variável threshold tem a função de ser a referência para separação da amostra em vários grupos, por isso ela deve representar da melhor forma possível, as condições socioeconômicas dos países, estados ou municípios.

A literatura relacionada aos testes de convergência da renda média per capita e aos testes de convergência na desigualdade de renda per capita, que utilizam o enfoque de clubes de convergência, estão longe do consenso quanto às possíveis candidatas à variável threshold. No entanto, a revisão dos principais trabalhos da área aponta para as três variáveis citadas anteriormente como indicadores básicos das condições socioeconômicas de qualquer economia ${ }^{5}$.

A Tabela 1 resume os resultados encontrados para a divisão da amostra entre os nós de decisão.

Os valores da estimativa do threshold foram respectivamente $0,57,72,44$ e 2,12, para as variáveis índice de Gini 1991, renda per capita 1991 e anos de escolaridade 1991. Baseada em 1000 repetições bootstrap, a hipótese nula de ausência de efeito threshold não pode ser rejeitada, independente da especificação (o teste do $p$ valor para este teste é maior que 0,05 em todos os casos). Dessa forma, não há clubes de convergência da desigualdade da renda per capita para os municípios cearenses, pelo menos quando se considera o modelo (6) e os anos 1991 e 2000.

\subsection{Convergência condicional da desigualdade de renda}

Uma vez que a hipótese de que a equação de convergência da desigualdade de renda seria não linear foi rejeitada, resta avaliar a hipótese alternativa, ou seja, a equação de convergência linear, considerando-se os possíveis determinantes da taxa de crescimento da desigualdade de renda no estado estacionário, o que leva à definição de convergência condicional.

A Tabela 2 apresenta os resultados das estimativas de Mínimos Quadrados Ordinários (MQO) do teste de convergência condicional da desigualdade de renda, para os municípios do Estado do Ceará.

0 coeficiente relacionado à variável logaritmo do índice de Gini do período inicial apresentou valor negativo e significativo ao nível de $1 \%$. Isso indica que o índice de Gini dos municípios do Estado do Ceará estaria convergindo para um valor médio condicional.

O valor médio de longo prazo do Índice de Gini, implicado pelo modelo econométrico, foi de aproximadamente 0,61 ; valor superior às médias do Índice de Gini nos anos $1991 \mathrm{e} 2000$, que foram iguais a 0,5241 e 0,5985 , respectivamente. Dessa forma, os municípios cearenses estariam convergindo em média para uma maior desigualdade de renda domiciliar per capita.

Tabela 1 - Resultados dos testes do efeito threshold para o modelo com Gini como medida de desigualdade de renda

\begin{tabular}{c|c|c|c|c}
\hline $\begin{array}{c}\text { Variável } \\
\text { dependente }\end{array}$ & $\begin{array}{c}\text { Variável } \\
\text { Threshold }\end{array}$ & $\begin{array}{c}\text { Estimativa } \\
\text { do threshold }\end{array}$ & $\begin{array}{c}\text { Teste } \\
\text { LM }\end{array}$ & p-valor \\
\hline \multirow{2}{*}{ Gini } & Indice de Gini 1991 & 0,5700 & 11,7127 & 0,2508 \\
\cline { 2 - 5 } & Renda per capita 1991 & 72,4400 & 12,0018 & 0,3420 \\
\cline { 2 - 5 } & Anos de estudo 1991 & 2,1200 & 12,5439 & 0,2560 \\
\hline
\end{tabular}

Nota: Hipótese nula: ausência de threshold.

Fonte: Elaborado pelos autores com base nos resultados da pesquisa. 
Tabela 2 - Teste de convergência da desigualdade de renda - Gini, 1991 e 2000

\begin{tabular}{|c|c|}
\hline & Gini \\
\hline \multirow{2}{*}{ Constante } & $-0,0460^{* * *}$ \\
\hline & $(0,0039)$ \\
\hline \multirow{2}{*}{ Log(Índice de Gini) ${ }_{1991}$} & $-0,0801^{* * *}$ \\
\hline & $(0,0052)$ \\
\hline \multirow{2}{*}{ Log(Renda do Trabalho) } & $-0,2159^{\star \star *}$ \\
\hline & $(0,0261)$ \\
\hline \multirow{2}{*}{ Log(Renda de Transferências) } & $-0,0580^{* *}$ \\
\hline & $(0,0292)$ \\
\hline \multirow{2}{*}{ Log(Anos de estudo) } & $0,0318^{\mathrm{NS}}$ \\
\hline & $(0,0424)$ \\
\hline \multirow{2}{*}{ Log(Proporção da População Adulta) } & $-0,0592^{\mathrm{NS}}$ \\
\hline & $(0,1963)$ \\
\hline $\mathbf{R}^{2}$ & 0.6766 \\
\hline Resíduo da Variância & 0,00004 \\
\hline Observações & 184 \\
\hline Longo Prazo(6) & 0,6125 \\
\hline
\end{tabular}

Nota: ${ }^{* * *}$ significativo a $1 \%,{ }^{* *}$ significativo a $5 \%$, ${ }^{\text {NS }}$ não significativo.

Fonte: Elaborado pelos autores.

Para visualizar esses resultados, 0 Gráfico 1 apresenta a Densidade de Kernel relativa ao índice de Gini para os anos 1991 e 2000, assim como uma linha vertical no valor médio do índice de Gini de longo prazo implicado pelo modelo (igual a 0,61250).

Como se pode observar, a distribuição estaria se movendo para a direita, em direção ao valor médio de longo prazo do índice de Gini. Em
1991, havia 16 (8,7\%) municípios cearenses com índice de Gini acima de 0,6125, com destaque para os municípios Brejo Santo, Mombaça e Cedro, que apresentaram os maiores valores desse índice. Em 2000, os municípios com valores de Gini acima do valor médio de longo prazo passaram para $82(44,56 \%)$, com os maiores valores de Gini encontrados nos municípios Mombaça, Amontada e Acopiara.

Gráfico 1 - Distribuição da desigualdade de renda dos municípios cearenses, medida pelo índice de Gini, 1991 e 2000

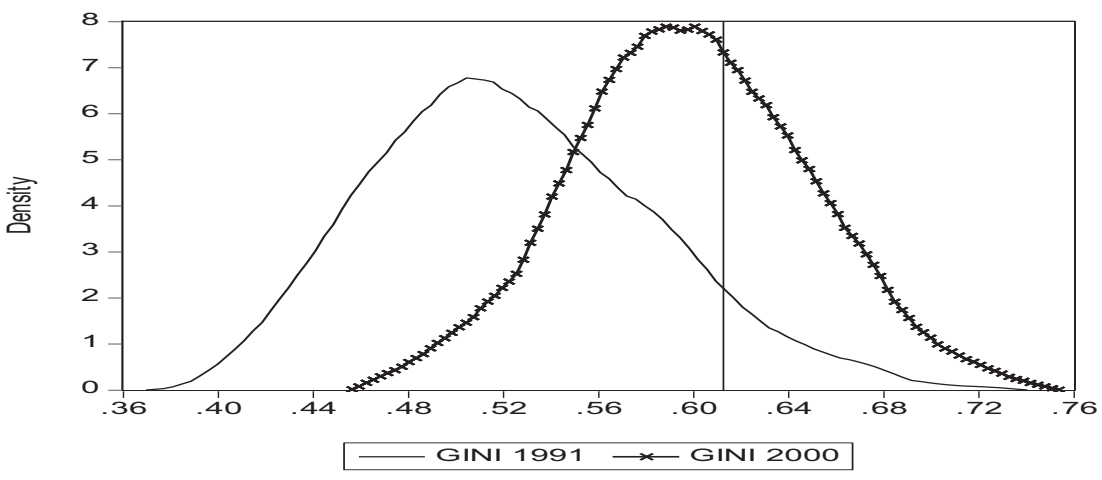

Fonte: Elaborado pelos autores com base nos dados da pesquisa. 
Esses resultados se assemelham aos encontrados no trabalho de Gomes (2007), que testou a convergência da desigualdade de renda per capita entre os municípios brasileiros. Gomes (2007) encontrou evidências desse tipo de convergência, sendo que o valor médio do índice de Gini de longo prazo implicado para os municípios da região Nordeste, identificada por meio de uma variável dummy, foi igual a 0,6050 . Esse valor seria uma espécie de valor médio de longo prazo dos estados que constituem a região Nordeste do Brasil e, desta forma, o índice de Gini de longo prazo, implicado para os municípios do estado do Ceará, estaria acima do valor médio da região. Ademais, 0 processo de convergência da desigualdade de renda nos municípios da região Nordeste, de acordo com Gomes (2007), também se dá com o deslocamento da distribuição do índice de Gini para a direita, em direção ao seu valor médio de longo prazo implicado pelo modelo, que se apresentou maior do que seu valor médio no ano 2000.

$\mathrm{Na}$ equação de convergência condicional da desigualdade de renda per capita entre os municípios cearenses, pode-se notar que tanto a renda do trabalho quanto a renda oriunda das transferências governamentais para as famílias foram significativas e importantes para explicar as taxas médias de crescimento da desigualdade de renda dos mesmos. 0 sinal negativo dos coeficientes associados àquelas variáveis indica uma relação inversa entre as mesmas e a taxa média de crescimento da desigualdade de renda. Nesse caso, um aumento na renda do trabalho e/ou na renda de transferências reduziria a taxa média de crescimento do índice de Gini, sendo que a elasticidade da renda do trabalho apresenta-se superior à elasticidade da renda das transferências governamentais.

Esses resultados corroboram os encontrados por Ferreira e Santos da Cruz (2008) em seu teste de convergência da desigualdade de renda para os estados brasileiros, considerando 0 enfoque de clubes de convergência. As estimativas desses autores mostram que existe uma diferença significativa, em termos absolutos, entre os valores da elasticidade da renda do trabalho e da elasticidade da renda das transferências governamentais, sendo essa diferença verificada para todos os clubes estimados. Em média, a elasticidade da renda do trabalho é cerca de quatro vezes maior do que a elasticidade das transferências governamentais.

As demais variáveis de controle, isto é, anos de escolaridade e percentual da população adulta, não foram significativas para explicar as taxas de crescimento do índice de Gini.

\subsection{Sensibilidade da convergência à medida de desigualdade de renda utilizada}

\subsubsection{Clubes de convergência da desigualdade de renda}

Ao considerar o índice de Gini como medida de desigualdade de renda, os testes de convergência realizados na seção anterior indicaram rejeição da hipótese de clubes de convergência nos municípios cearenses, mas apontaram um processo de convergência condicional para o valor médio da desigualdade de renda desses municípios, com 0 índice de Gini de longo prazo sinalizando para um aumento no valor médio da desigualdade de renda.

No entanto, como uma medida de desigualdade de renda é uma forma de agregar em um único indicador diferenças de renda de milhões de pessoas, não é surpreendente, portanto, que existam diversas formas alternativas de medir a desigualdade de renda (INSTITUTO DE PESQUISA ECONÔMICA APLICADA, 2006). Dessa forma, é natural questionar se essas evidências são sustentadas quando se consideram medidas alternativas de desigualdade de renda.

Para examinar essa questão foram realizados alguns testes de significância estatística do modelo threshold em relação à especificação linear, considerando-se medidas alternativas de desigualdade de renda.

Os valores do teste do LM para essas medidas, considerando-se, novamente, diferentes medidas para a variável threshold, estão apresentados na Tabela 3. 
Tabela 3 - Resultados dos testes do efeito threshold para os modelos com medidas alternativas de desigualdades de renda

\begin{tabular}{|c|c|c|c|c|}
\hline $\begin{array}{l}\text { Variável } \\
\text { depend ente }\end{array}$ & $\begin{array}{l}\text { Variaivel } \\
\text { threshold }\end{array}$ & $\begin{array}{c}\text { Estimativ a do } \\
\text { threshold }\end{array}$ & $\begin{array}{c}\text { Teste } \\
\text { LM }\end{array}$ & p-ralor \\
\hline \multirow{3}{*}{ Theil - L } & Indice de Theil - L 1991 & 0.6000 & 10,0299 & 0,5270 \\
\hline & Renda per capita 1991 & 54,6000 & 15,0061 & 0,0960 \\
\hline & Anos de estudo 1991 & 2,1200 & 14,0259 & 0,1160 \\
\hline \multirow{3}{*}{$\begin{array}{c}\text { Razão } \\
10 \%+/ 40 \%-\end{array}$} & Raż̃o $10 \%+40 \%$ - 1991 & 19,7600 & 10,1881 & 0,5420 \\
\hline & Renda per capita 1991 & 42,8400 & 12,8763 & 0,2240 \\
\hline & Anos de estudo 1991 & 1,3100 & 12,2506 & 0,2870 \\
\hline \multirow{3}{*}{$\begin{array}{c}\text { Razão } \\
20 \%+/ 40 \% \text { - }\end{array}$} & Raż̃o $20 \%+/ 40 \%$ - 1991 & 12,6200 & 9,9628 & 0,5400 \\
\hline & Renda per capita 1991 & 42,8400 & 11,7164 & 0,3340 \\
\hline & Anos de estudo 1991 & 1,3100 & 13,3289 & 0,1750 \\
\hline
\end{tabular}

Nota: Hipótese nula: ausência de Threshold.

Fonte: Elaborado pelos autores.

Baseada em 1000 repetições bootstrap, a hipótese nula de ausência de efeito threshold não pode ser rejeitada, independente da especificação (os p-valores para cada variável threshold foi superior a $0,05 \mathrm{em}$ todos os casos). Esses resultados reforçam as evidências encontradas na seção anterior, ou seja, não se verificam clubes de convergência da desigualdade de renda nos municípios cearenses, quando se considera o modelo (7) e os anos 1991 e 2000.

\subsubsection{Convergência condicional da desigualdade de renda}

Novamente, dado que a hipótese de que a equação de convergência da desigualdade de renda seria não linear foi rejeitada, partiu-se para testar a hipótese alternativa, ou seja, a equação de convergência linear.

A Tabela 4 apresenta os resultados das estimativas de MQO do teste de convergência condicional da desigualdade de renda entre os municípios cearenses, considerando-se medidas alternativas de desigualdade de renda.

Os coeficientes relacionados às variáveis logaritmo do índice de Theil, logaritmo da Razão $10 \%+/ 40 \%$ - e logaritmo da Razão $20 \%+/ 40 \%$, medidos no período inicial, apresentaram valores negativos, significativos ao nível de $1 \%$ e próximos ao valor encontrado pelo índice de Gini ${ }^{8}$. De acordo com as equações empíricas de convergência nos modelos neoclássicos de crescimento econômico, sinais negativos e significativos desse parâmetro indicam um processo de convergência para o valor médio das medidas de desigualdade de renda. Logo, essas estimativas reforçam os resultados encontrados na seção anterior para 0 índice de Gini.

Os valores médios de equilíbrio de longo prazo para o índice de Theil - L, Razão $10 \%+/ 40 \%$ e Razão $20 \%+140 \%$-, implicado pelos modelos econométricos, foram iguais a $0,6425,22,4019$ e 14,5154 , respectivamente. 0 valor médio implicado do índice de Theil $-L$ foi superior à média do índice de Theil $-L$ nos anos 1991 e 2000, que foram iguais a 0,4933 e 0,5873 , respectivamente. Os valores médios implicados das duas razões entre a renda dos mais ricos e dos mais pobres foram maiores que os valores médios dessas variáveis no ano 1991, mas menores que no ano 2000 (o valor médio da Razão 10\%+/40\%- em 1991 foi 15,425, e em 2000 foi 33,859 , quanto à Razão $10 \%+/ 40 \%$ - esses valores foram 10,377 e 22,397). 
Tabela 4 - Teste de convergência da desigualdade de renda - medidas alternativas de desigualdade, 1991 e 2000

\begin{tabular}{|c|c|c|c|}
\hline & Theil - L & $\begin{array}{c}\text { Razão } \\
10 \%+/ 40 \%=\end{array}$ & Razão $20 \%+/ 40 \%$ - \\
\hline \multirow[t]{2}{*}{ Constante } & $-0,0388^{* * *}$ & $0,1769^{* * *}$ & $0,1396^{* * *}$ \\
\hline & $(0,0069)$ & $(0,0349)$ & $(0,0331)$ \\
\hline \multirow[t]{2}{*}{ log(Índice de Theil - L) 1991} & $-0,0803^{* * *}$ & - & - \\
\hline & $(0,0052)$ & - & - \\
\hline \multirow[t]{2}{*}{$\log (\text { Razão 10\%+/40\%-) })_{1991}$} & - & $-0,0718^{* * *}$ & - \\
\hline & - & $(0,0098)$ & - \\
\hline \multirow[t]{2}{*}{$\log ($ Razão 20\%+/40\%-) 1991} & - & - & $-0,0692^{* * *}$ \\
\hline & - & - & $(0,0108)$ \\
\hline \multirow[t]{2}{*}{ Log(Renda do Trabalho) } & $-0,0195^{\mathrm{NS}}$ & $-1,8892^{* * *}$ & $-1,8977^{* * *}$ \\
\hline & $(0,0625)$ & $(0,1860)$ & $(0,1763)$ \\
\hline \multirow[t]{2}{*}{ Log(Renda de Transferências) } & $-0,0425^{\mathrm{NS}}$ & $-0,4237^{* * *}$ & $-0,3887^{* * *}$ \\
\hline & $(0,0777)$ & $(0,1612)$ & $(0,1510)$ \\
\hline \multirow[t]{2}{*}{ Log(Anos de estudo) } & $0,0354^{\mathrm{NS}}$ & $0,4804^{\mathrm{NS}}$ & $0,5071^{* *}$ \\
\hline & $(0,1057)$ & $(0,2675)$ & $(0,2561)$ \\
\hline \multirow[t]{2}{*}{ Log(Percentual da População Adulta) } & $-0,1203^{N S}$ & $-0,2379^{N S}$ & $-0,2502^{\text {NS }}$ \\
\hline & $(0,4471)$ & $(1,1836)$ & $(1,1112)$ \\
\hline $\mathbf{R}^{2}$ & 0.6098 & 0.5906 & 0.6033 \\
\hline Resíduo da Variância & 0,0002 & 0.0013 & 0,0012 \\
\hline Observações & 184 & 184 & 184 \\
\hline Longo Prazo ${ }^{7}$ & 0,6425 & 22,4019 & 14,5154 \\
\hline
\end{tabular}

Nota: ${ }^{* * *}$ significativo a $1 \%,{ }^{* *}$ significativo a $5 \%$, Ns não significativo.

Fonte: Elaborado pelos autores.

Novamente, para visualizar esses resultados, o Gráfico 2 mostra a Densidade de Kernel relativa ao índice de Theil (painel a), Razão 10\%+/40\%- (painel b) e Razão 20\%+/40\%- (painel c), para os anos 1991 e 2000.

Em cada um desses painéis, apresenta-se também uma linha vertical no valor médio de longo prazo implicado para cada uma dessas medidas de desigualdade. $O$ índice de Theil - $L$ apresentou uma evolução semelhante ao índice de Gini, ou seja, sua distribuição está se movendo para a direita, em direção ao seu valor médio de equilíbrio de longo prazo. No que diz respeito às variáveis Razão 10\%+/40\%- e Razão 20\%+/40\%-, a evolução das mesmas se mostrou inversa à dos índices de Gini e Theil, isto é, elas estão convergindo para valores médios de longo prazo que são menores do que aqueles observados no ano de 2000.

Se for considerado o valor médio de equilíbrio de longo prazo implicado para o índice de Theil-L, há uma sinalização para um aumento na desigualdade de renda, assim como no índice de Gini. No entanto, o valor médio de equilíbrio de longo prazo das razões entre as rendas dos mais ricos e dos mais pobres indicam que os municípios cearenses estariam convergindo em média para uma menor desigualdade de renda domiciliar per capita. Esses resultados podem ser um indicativo de que o aumento esperado na desigualdade de renda domiciliar per capita, nos municípios cearenses, seria influenciado mais pela desigualdade dentro de cada classe do que pela desigualdade entre as classes. 


\section{Gráfico 2 - Distribuição das medidas alternativas de desigualdade de renda dos municípios cearenses, 1991 e 2000}

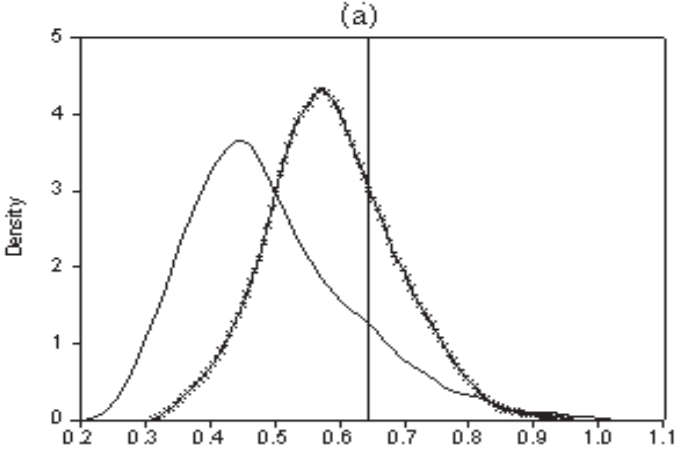

(b)

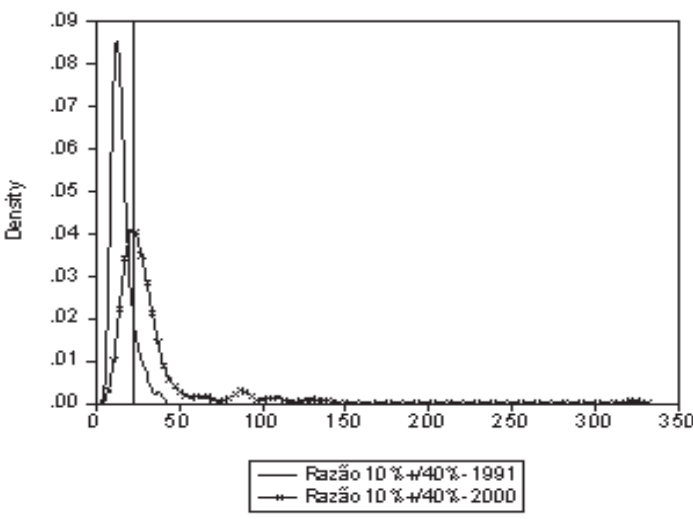

Fonte: Elaborado pelos autores.

Com exceção do modelo de convergência da desigualdade de renda medido pelo índice de Theil - $\mathrm{L}$, os demais mostraram que a renda do trabalho $\mathrm{e}$ a renda oriunda das transferências governamentais para as famílias foram significativas e importantes para explicar as taxas médias de crescimento da desigualdade de renda. Novamente, o sinal negativo do coeficiente relacionado a essas variáveis indica uma relação inversa entre as mesmas e as taxas médias de crescimento da desigualdade de renda, sendo que, novamente, a elasticidade da renda do trabalho apresentou-se superior à elasticidade da renda das transferências governamentais para ambos os modelos nos quais esses coeficientes foram significativos.

Por fim, vale destacar que a taxa de crescimento da média dos anos de estudo da população com 25 anos ou mais foi significativa (c)

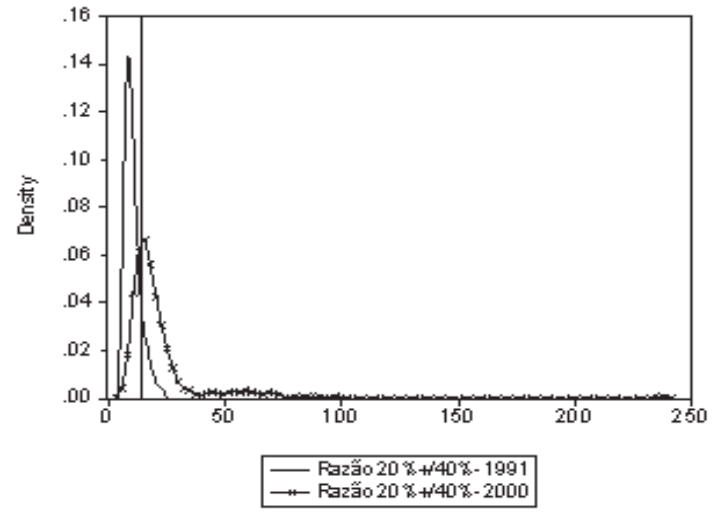

para explicar as taxas médias de crescimento da desigualdade de renda nos municípios cearenses, ao longo do período de análise, quando se considerou a Razão $20 \%+/ 40 \%$ - como medida de desigualdade. Apesar de significativo, o sinal do coeficiente associado a essa variável apresentou-se como positivo, contrário ao esperado, uma vez que maiores taxas de crescimento dos anos médios de escolaridade de parte considerável da população economicamente ativa, ou seja, pessoas com 25 anos ou mais, deveria reduzir a desigualdade, em decorrência de sua externalidade positiva, isto é, sua capacidade de ampliar a produtividade do trabalho.

\section{CONCLUSÃO}

Este trabalho investigou a hipótese de convergência da desigualdade de renda entre os 
municípios cearenses. As estimativas indicaram que não existem diferentes regimes de convergência (Clubes de Convergência) da desigualdade de renda nos municípios cearenses, considerando-se os anos 1991 e 2000. No entanto, parece existir uma evidência de que esses municípios estariam em um processo de convergência condicional, no qual o nível médio de equilíbrio de longo prazo da desigualdade da renda domiciliar per capita seria maior do que o valor médio do Gini de 2000. Portanto, poder-se-ia esperar um aumento médio da desigualdade de renda dentro dos municípios cearenses, caso fossem mantidas as mesmas condições econômicas que nos períodos de análise.

Como a renda proveniente do trabalho apresentou elasticidade superior ao da renda de transferências governamentais, sendo que ambas apresentaram correlação negativa com a taxa de crescimento do Gini, calculada no período de 1991 a 2000, seria de se esperar que políticas que favoreçam o acesso ao mercado de trabalho e elevem a produtividade do trabalhador produzam resultados mais satisfatórios do que políticas enfatizadas apenas em transferências governamentais.

Por fim, deve-se ressaltar que há duas direções para a realização de trabalhos futuros sobre convergência da desigualdade de renda. Em primeiro lugar, é necessário explicar melhor por que esse tipo de convergência estaria ocorrendo. Nesse caso, na esfera de países, poder-se-ia recorrer à hipótese de Ravallion (2001), de que essa convergência surge, possivelmente, da interação da convergência das políticas econômicas entre os mesmos no âmbito da desigualdade. A transição generalizada para uma economia mais orientada para o mercado, por exemplo, pode atenuar extremos na desigualdade dentro dos países, mas é limitada, em decorrência das diferenças relacionadas à distribuição de ativos entre os países. Na esfera das regiões, estados ou municípios de um mesmo país, por sua vez, podese conjeturar que o processo de convergência da desigualdade de renda esteja ocorrendo devido à ampliação das políticas de transferências de renda, bem como por uma provável convergência nas distribuições de recursos humanos e físicos.

Em segundo lugar faz-se necessário produzir mais evidências empíricas sobre a convergência da desigualdade de renda entre os municípios cearenses, para que se possa ter uma ideia mais robusta da ocorrência ou não desse processo. Isso pode ser feito tanto por meio da utilização de métodos alternativos de estimação, como por exemplo, métodos não paramétricos, bem como por meio de modelos mais elaborados que incluam outras variáveis explicativas relevantes para entender a evolução da desigualdade.

\section{REFERÊNCIAS}

AYAGARI, R. Uninsured Idiosyncratic Risk and Aggregate Savings. Quarterly Journal of Economics, Oxford, n. 109, p. 659-684, 1994.

BARRETO, R. S. C. Desenvolvimento regional e convergência de renda nos municípios do Estado do Ceará. Viçosa: UFV, 2007. 191 f. Tese (Doutorado em Economia Aplicada) - Universidade Federal de Viçosa, 2007.

BÉNABOU, R. Inequality and growth. In: BERNANKE, Ben S.; ROTEMBERG, Julio J. (Eds.). NBER Macro Annual, Cambridge, Mass.: MIT Press, 1996. v. 11.

BERTOLA, G. Accumulation and Extent of Inequality, CEPR Discussion Paper, London, 1187, 1995.

BLEANEY, M.; NISHIYAMA, A. Convergence in inequality: differences between advanced and developing countries. Economics Bulletin, [S. I.], v. 4, p. 1-10, 2003.

DURLAUF, S. N.; JOHNSON, P. A. Multiple regimes and cross-country growth behavior. Journal of Applied Econometrics, Canada, v. 10, n. 4, p. 36584, 1995.

EZCURRA, R.; PASCUAL, P. Is there convergence in income inequality levels among the European regions? Applied Economics Letters, [S. I.], v. 12, n. 12, p. 763-766, 2005. 
FERREIRA, R. T.; SANTOS DA CRUZ, M. Clubes de convergência na desigualdade de renda nos municípios brasileiros. In: ENCONTRO NACIONAL DA ANPEC, 36., 2008, Salvador. Anais... Salvador, 2008.

GALOR, O. Convergence? Inferences from Theoretical Models. The Economic Journal, [S. I.], v. 106, p. 1056-1069, 1996.

GOMES, F. Convergence in income inequality: the case of Brazilian municipalities. Economics Bulletin, [S. I.], v. 15, n. 15, p. 1-19, 2007.

HANSEN, B. E. Sample splitting and threshold estimation. Econometrica, New York, v. 68, n. 3, p. 575-603, 2000.

INSTITUTO BRASILEIRO DE GEOGRAFIA E ESTATÍSTICA. Nota Técnica. Brasília, DF, 2011.

INSTITUTO DE PESQUISA E ESTRATÉGICA ECONÔMICA DO CEARÁ. O Ceará em Números. Fortaleza, 2010.

INSTITUTO DE PESQUISA ECONÔMICA APLICADA. Sobre a recente queda na desigualdade de renda no Brasil: nota técnica. Brasília, DF, 2006.

MAYORGA, R. D.; TABOSA, F. J. S.; AMARAL FILHO, J. Análise de desigualdade de renda no estado do Ceará. In: CONGRESSO DA SOCIEDADE BRASILEIRA DE ECONOMIA, ADMINISTRAÇÃO E SOCIOLOGIA RURAL, 45., 2007, Londrina, PR. Anais... Londrina, PR: SOBER, 2007. CDROM.

OLIVEIRA JÚNIOR, J. N. et al. Determinação dos clubes de convergência da renda per capita agrícola - uma análise para os municípios cearenses. Revista de Economia e Sociologia Rural, Piracicaba, SP, v. 47, n. 4, p. 995-1021, 2009.

PANIZZA, U. Convergence in income inequality. Journal of Income Distribution, Canada, v. 10, p. 5-12, 2001.

PONTES, D. O.; ARRAES, R. A.; PENA, C. Crescimento econômico e desigualdade de renda no Ceará. In: CARVALHO, E. B. S. (Org.). Economia do Ceará em Debate. Fortaleza: IPECE, 2010. p. 102130.
PROGRAMA DAS NAÇÕES UNIDAS PARA O DESENVOLVIMENTO; INSTITUTO DE ECONOMIA APLICADA; FUNDAÇÃO JOÃO PINHEIRO. Atlas de desenvolvimento humano do Brasil. Rio de Janeiro, 2003.

QUAH, D. Empirics for economic growth and convergence. European Economic Review, [S. I.], v. 40, p. 1353-1375, 1996.

RAVALLION, M. Inequality convergence. Economics Letters, [S. I.], v. 80, p. 351-361, 2003.

Inequality convergence. Policy Working Paper, [S. I.], n. 2645, 2001.

SILVA, V. H. O.; LOUREIRO, A. O. F. A distribuição de renda no estado do Ceará no período 20012005: nota técnica $n^{0}$. 25. Fortaleza: IPECE, 2007. (Textos para Discussão)

SOARES, W. R. F. Desigualdade de renda no Ceará: uma análise a partir do emprego formal. In: ENCONTRO NACIONAL DE ESTUDOS POPULACIONAIS, 16., 2008, Caxambu, MG. Anais... Caxambu, MG: ABEP, 2008. CDROM.

SOUSA, E. P. Desenvolvimento socioeconômico e hierarquização dos municípios do estado do Ceará: uma análise multivariada. Revista Economia em Debate (URCA), Crato, CE, v. 3, p. 107-130, 2009.

NOTAS

1 É importante observar que existem trabalhos que testaram a hipótese de convergência da renda média entre os municípios do Ceará. Barreto (2007), Oliveira Júnior e outros (2009) e de Pontes, Arraes e Pena (2010), por exemplo, buscaram testar a convergência da renda per capita entre os municípios cearenses, sendo que o foco do primeiro consistiu em verificar se houve convergência e transbordamento espacial no período de 1996 a 2003, utilizando como método analítico a Econometria Espacial; o segundo buscou determinar os clubes de convergência da renda per capita agrícola, empregando um modelo com efeito threshold, para o período de 1970 a 1996; e o terceiro buscou testar a convergência da renda per capita entre os municípios e os determinantes da desigualdade de renda com base na decomposição do índice de Theil no período de 1995 a 2007.

${ }^{2}$ Essa equação ficou conhecida na literatura de convergência como Equação de Barro, devido ao economista David 
Barro, que foi um dos primeiros a propô-la. Apesar de muito utilizada, a Equação de Barro não está livre de críticas. Uma das principais críticas à utilização dessa equação como teste de convergência para renda média per capita foi feita por Quah (1993). Segundo esse autor, como o coeficiente estimado representa um valor médio da amostra utilizada, esse experimento pode na verdade não estar representando de forma clara sua real tendência. Dessa forma, os resultados dessas equações estariam sujeitos à chamada Falácia de Galton, expressão que se refere aos estudos originais de Francis Galton, que fez uma inferência incorreta a partir de suas observações. A falácia ocorre porque a seleção de observações tomando seus extremos levaria naturalmente a essa tendência.

${ }^{3}$ Bénabou (1996) e Ravallion (2001; 2003) testaram a hipótese da convergência da desigualdade de renda per capita para diferentes amostras cross section de países, por meio de uma equação com a taxa de crescimento do índice de Gini como variável dependente, em detrimento ao logaritmo do índice de Gini no período inicial. Ferreira e Santos da Cruz (2008), por sua vez, estenderam os modelos de Bénabou (1996) e Ravallion (2001; 2003), incluindo mais três variáveis explicativas (renda do trabalho, renda das transferências governamentais e anos de escolaridade da população com idade igual ou superior a 25 anos) e uma função indicadora para capturar possíveis clubes de convergência.

${ }^{4}$ Apesar da equação (7) indicar a presença de apenas dois clubes ou regimes de convergência, o teste de não linearidade, ou efeito threshold, é aplicado de forma iterativa até não haver mais evidências estatísticas da necessidade de novas subdivisões amostrais.

${ }^{5}$ Durlauf e Johnson (1995), por exemplo, utilizaram as variáveis renda e escolaridade, medidas no período inicial, para verificar a existência de múltiplos regimes de convergência de renda média per capita para uma amostra de países. Bénabou (1996), Ravallion (2003), Bleaney e Nishiyama (2003), entre outros, utilizaram a variável Gini medida no período inicial como possível variável threshold. A utilização de medidas alternativas para a variável threshold, como feito neste trabalho, permite verificar a robustez das estimativas. Vale observar que apenas as variáveis renda per capita, em 1991, e anos de estudo, em 1991, apresentaram elevado grau de correlação, aproximadamente 0,84 .

${ }^{6}$ No modelo de crescimento neoclássico com crescimento populacional e sem progresso tecnológico, as variáveis per capita são constantes no estado estacionário. No entanto, as variáveis em nível crescem à mesma taxa que o crescimento populacional. Neste caso, o valor do equilibrio de longo-prazo do índice de Gini implicado pela equação (6) é dado por $\exp \left[\left(-\left(\beta_{0}\right) /\left(\beta_{1}\right)\right)-\left(\left(\beta_{2+} \beta_{3+} \beta_{4+} \beta_{5}\right)\left[n /\left(\beta_{1}\right)\right)\right]\right.$, em que $n$ é média da taxa média geométrica de crescimento anual da população residente no Estado do Ceará, para o período de 1950 a 2010, que de acordo com dados do IBGE foi igual 2,24\%.
${ }^{8} \mathrm{~A}$ proximidade entre os valores do coeficiente $\beta$ nos diferentes modelos deve-se à elevada correlação entre as diferentes medidas de desigualdade de renda.

\section{Airton Lopes Amorim}

Economista

Mestre em Economia pela Universidade Federal de Viçosa (UFV)

E-mail: aimorim2007@gmail.com

\section{Ricardo Bruno Nascimento dos Santos}

Economista

Doutor em Economia pela Universidade Federal de Viçosa (UFV)

Professor da Universidade Federal do Pará (UFPA)

E-mail: ricardobns@gmail.com

\section{Eliane Pinheiro de Sousa}

Economista

Doutora em Economia pela Universidade Federal de Viçosa (UFV)

Professora da Universidade Regional do Cariri (URCA)

E-mail: pinheiroeliane@hotmail.com

\section{Daniel Arruda Coronel}

Economista

Doutor em Economia pela Universidade Federal de Viçosa (UFV)

Professor Adjunto da Universidade Federal de Santa Maria (UFSM)

E-mail: daniel.coronel@uol.com.br

\section{Universidade de Federal de Viçosa - UFV}

Aveinda Peter Henry Rolfs, s/n, Viçosa/MG CEP: $36570-000$

Universidade Federal do Pará - UFPA

Rua Augusto Corrêa, 01 - Guamá, Belém - PA CEP: 66075-110

Universidade Regional do Cariri - URCA

Rua Coronel Antonio Luiz, no 1161- Pimenta, Crato - CE, CEP: $63105-000$

Universidade Federal de Santa Maria - UFSM

Prédio 74C, Térreo, Sala 4112 - Santa Maria, RS CEP: 97105900

${ }^{7}$ Ver nota de rodapé 2. 